\title{
Lateral instability and tunnel erosion of a submarine pipeline: competition mechanism
}

\author{
Yu-min Shi ${ }^{1,2} \cdot$ Fu-ping Gao ${ }^{1,2}$
}

Received: 22 January 2017 / Accepted: 11 May 2017

(C) Springer-Verlag Berlin Heidelberg 2017

\begin{abstract}
In submarine geological and hydrodynamic environments, either tunnel erosion or lateral instability could be initiated where there is a shallowly embedded pipeline. Unlike previous studies on the tunnel erosion of sand and the lateral instability of pipelines, in this study we performed correlation analyses on the competition mechanism for these two physical processes. By correlating the critical flow velocities of these two processes, the instability envelope for the pipe-soil interaction system is established, which can be described using three key parameters: the embedment-to-diameter ratio, the dimensionless submerged weight of the pipe, and the corresponding critical flow velocity. The analysis procedure is further proposed to first determine the instability mechanism and then the critical velocity of ocean currents. Our parametric study indicates that tunnel erosion is more prone to emerging than lateral instability with small embedment-to-diameter ratio values. With increasing pipeline embedment, tunnel erosion can be suppressed and lateral instability therefore occurs more frequently. Moreover, for light pipelines, lateral instability is more likely to be triggered than tunnel erosion.
\end{abstract}

Fu-ping Gao

fpgao@imech.ac.cn

Yu-min Shi

shiyumin@imech.ac.cn

1 Key Laboratory for Mechanics in Fluid Solid Coupling Systems, Institute of Mechanics, Chinese Academy of Sciences, Beijing 100190, China

2 School of Engineering Science, University of Chinese Academy of Sciences, Beijing 100049, China
Keywords Submarine pipeline $\cdot$ Pipeline-soil interaction system $\cdot$ Lateral instability $\cdot$ Tunnel erosion $\cdot$ Competition mechanism

\section{Introduction}

As the offshore exploitation of gas and oil moves into deeper waters, ocean currents become the prevailing hydrodynamic load on submarine pipelines. Meanwhile, the preference now is for submarine pipelines in deeper waters to be laid directly on the seabed with a small embedment. In ocean currents, the flow over the pipeline and the seepage flow inside the underlying soil can be generated synchronously for complex flowpipeline-soil interactions (see Fig. 1). Two physical processes or phenomena, i.e., tunnel erosion and lateral instability, can be involved, which may pose risks for the safety and integrity of submarine pipelines during their operational cycles (see Fredsøe 2016). Pipeline spanning resulting from tunnel erosion may cause further structural vibrations in the pipeline, i.e., vortex-induced vibrations (Det Norske Veritas 2006). Lateral instability can, however, promote the development of global buckling of pipelines (Det Norske Veritas 2010).

Over the past a few decades, complex flow-pipeline-soil interactions have received much attention among researchers for revealing their underlying mechanisms. It should be noted that the tunnel erosion of sand and lateral instability of pipelines have been investigated mainly in relation to the aspects of hydrodynamics and geotechnics, respectively, which are reviewed briefly as follows.

\section{(1) Tunnel erosion of sand}

For a pipeline laid on an erodible seabed, if the hydrodynamics on a shallowly embedded pipeline are sufficiently 
Fig. 1 Illustration of the flowpipeline-soil interaction system in ocean currents

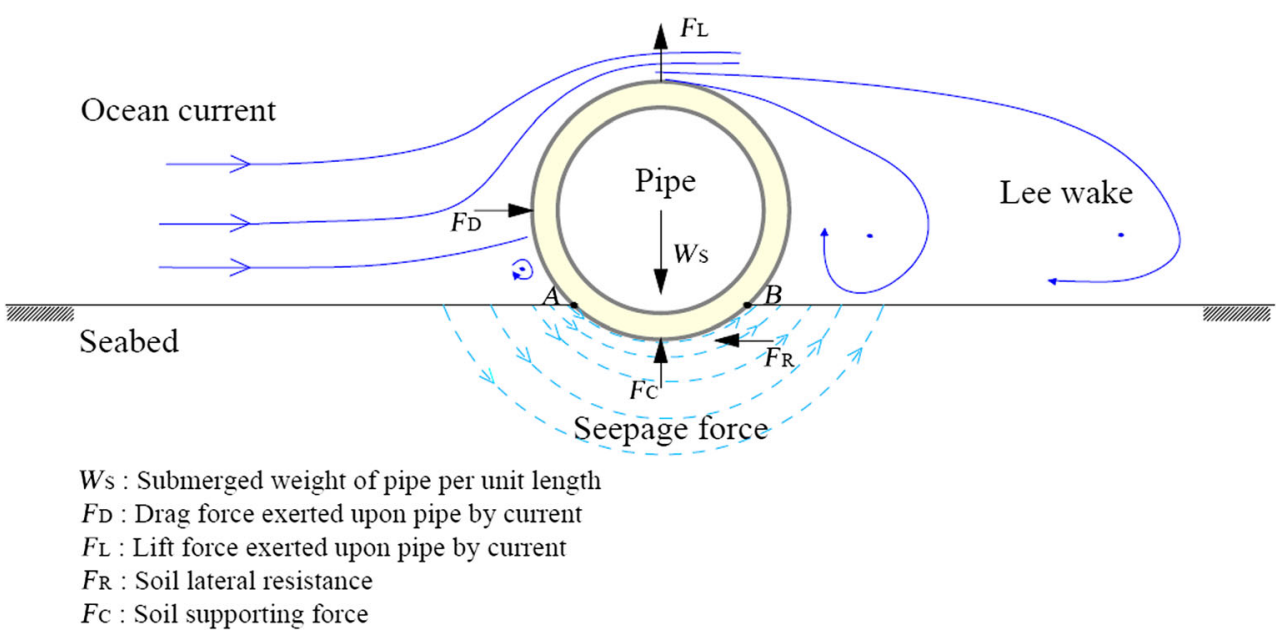

strong, tunnel erosion underneath the pipeline can be initiated. As a special pattern of local scour around marine structures, the tunnel erosion is usually characterized by substantial sediments below a shallowly foundation (e.g., the pipeline) being rapidly washed away, followed by a complete spanning of the structure (see Sumer and Fredsøe 2002).

To reveal the mechanism for tunnel erosion, the flow visualization in water flume was utilized for physical modeling of the flow-pipe-soil interaction. As described by Mao (1988), lee-wake vortices around the pipeline in steady currents can cause a pressure drop between the upstream and downstream of the pipe, which would result in further seepage flow within the seabed. The tunnel erosion involves a coupling process between the flow field above and the seepage field below the pipe (see Fig. 1). In the flume tests by Chiew (1990), the tunnel erosion was effectively suppressed once an impermeable plate was placed at the upstream of the pipe to reduce the seepage gradient, indicating that seepage force plays a key role in the occurrence of tunnel erosion. In later flume tests by Sumer et al. (2001), pressure gradients were measured and found to be significantly affected by the flow velocity and burial depth of the pipeline. The piping process was also captured below the pipeline when the flow velocity is increased gradually due to excessive seepage force. A series of flume observations by Gao et al. (2007a) indicated that, during the process of the pipeline being suspended, three characteristic stages usually exist (see Fig. 2a), i.e., stage I: local scour; stage II: onset of tunnel erosion; and stage III: complete suspension of the pipe. That is, with increasing flow velocity, local scour around pipeline emerged first (stage I); the seepage failure underneath the pipeline essentially triggered tunnel erosion (stage II); and the pipeline was finally suspended completely (stage III). As discussed by Gao and Luo (2010), the local scour could coexist during the process of tunnel erosion, but the former (which brings about the seabed profile change) usually develops much more slowly than the latter.
As mentioned earlier, seepage failure resulting from a pressure drop has been recognized as the dominant cause of the onset of tunnel erosion. A flow-pipe-seepage sequential coupling FEM model was proposed by Gao and Luo (2010) for implementing the coupling between water flow field and soil seepage field. The coupling analysis indicated that the maximum hydraulic gradient in the underlying soil always appears at the downstream intersection of the pipeline with the soil surface, i.e., the exit of seepage flow (see point B in Fig. 1). Such numerical simulation was also consistent with the experimental observations of Sumer et al. (2001).

To quantitatively predict the onset of tunnel erosion in steady currents, the criterion for seepage failure should be well-established and chosen for numerical simulations. The conventional hydraulic gradient for seepage failure (upward), i.e., $i_{\mathrm{cr} 0}=(1-n)(s-1)$ were adopted in some previous analyses (e.g., Sumer et al. 2001; Zang et al. 2009; Zhang et al. 2016), where $n$ is the porosity of sand and $s$ is the specific gravity of sand grains. Thus, the direction of seepage flow at the exit is tangent to the pipe surface instead of upward vertically; the critical hydraulic gradient for the oblique seepage failure of sand was derived by Gao and Luo (2010): $i_{\mathrm{cr}}=(\sin \theta+\cos \theta \tan \varphi)(1-n)(s-1)$, where $\theta$ is the half angle of pipe embedment and $\varphi$ is the internal friction angle of sand. Furthermore, in their study, the non-dimensional flow velocity for the onset of tunnel erosion was established:

$\theta_{\mathrm{cr}} \approx 2.51+0.068 \varphi \quad(0<e / D<0.25)$

where

$\theta_{\mathrm{cr}}=\frac{U_{\mathrm{CT}^{2}}}{e g(1-n)\left(s^{-1}\right)}$

$\theta_{\text {cr }}$ can be termed as the revised Shields number for tunnel erosion underneath of the pipeline with initial embedment $e$; $U_{\mathrm{CT}}$ is the critical flow velocity for the onset of tunnel erosion; $g$ is the gravitational acceleration; $D$ is the pipe diameter. Note 
Fig. 2 Previous observations for typical stages in the two physical processes: (a) tunnel erosion of sand (Gao et al. 2007b); (b) lateral instability of pipeline (Gao et al. 2002)

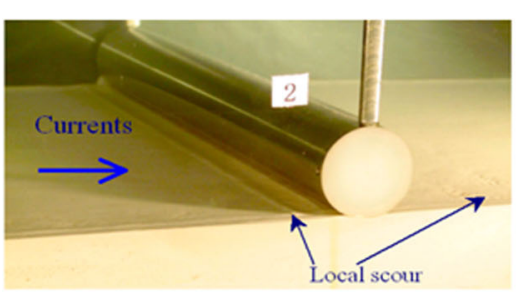

(I): Local scour

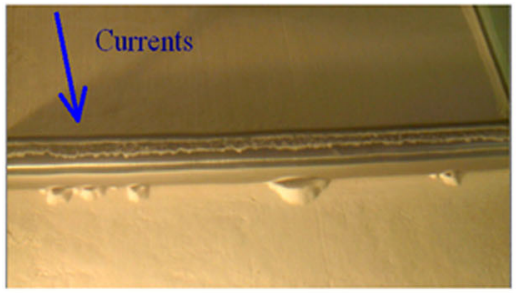

(II): Onset of tunnel erosion

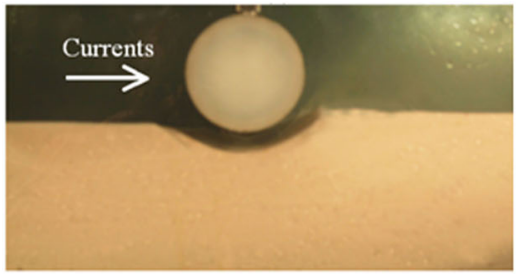

(III): Complete suspension of pipe

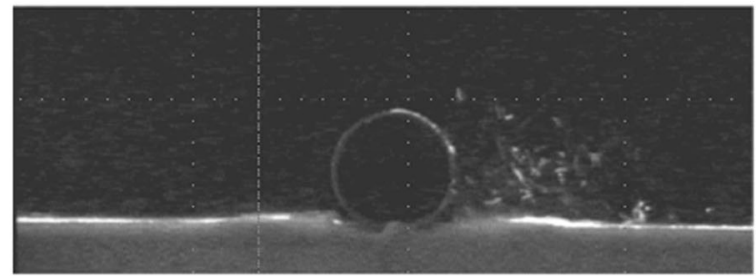

(I): Onset of sand scour

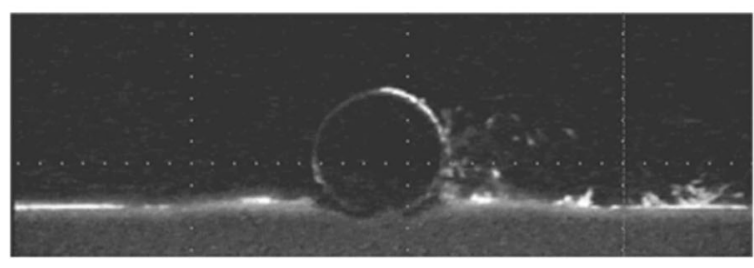

(II): Pipe moves slightly

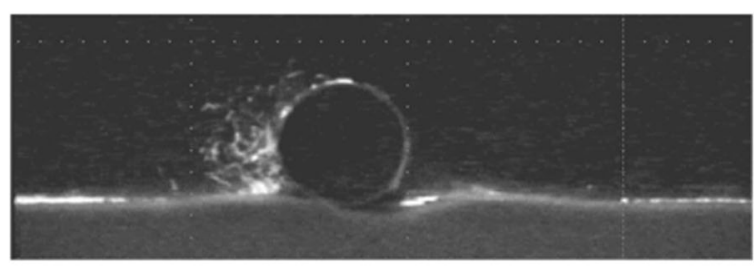

(III): Pipe breakouts that in the updated relationship by Eq. 1, the critical flow is correlated with the internal friction angle of sand and the porosity of sand for the conditions of shallow embedment of pipelines.

\section{(2) Lateral instability of pipe}

Due to the action of ocean currents, the partially embedded pipeline is subjected to hydrodynamic forces, including the horizontal drag force $F_{\mathrm{D}}$ and the vertical lift force $F_{\mathrm{L}}$ (see Fig. 1). When the horizontal drag force becomes severe enough to exceed the lateral soil resistance $\left(F_{\mathrm{R}}\right)$, the lateral instability of the pipeline would be triggered (see Gao et al. 2002).

An accurate estimation of soil lateral resistance is crucial for the design of on-bottom pipeline stability (Det Norske Veritas 2010). Experiments on pipe-soil interaction with a mechanical actuator (Lyons 1973) indicated that the soil resistance to the pipeline is far beyond that described with conventional Coulomb friction theory. Based on the results of mechanical-actuator loading tests, an empirical pipe-soil interaction model was proposed (Wagner et al. 1989), in which the lateral resistance $F_{\mathrm{R}}$ was regarded as the scalar sum of two components: sliding resistance and passive soil pressure. For lateral movement with large amplitude, the influence of soil berm accumulated in the proximity of the pipe would be taken into account to evaluate pipe-soil interactions (White and Cheuk 2008; Youssef et al. 2013; Wang and Liu 2016).
In reality, the ocean current and waves exert hydrodynamic loads not only on the pipeline but also on the soil. As such, the lateral instability of a shallowly embedded pipeline involves complex flow-pipe-soil interactions. In the process of a pipeline losing stability, three characteristic stages were observed experimentally under waves and steady currents (Gao et al., 2002, 2007a): (I) onset of local scour; (II) pipe rocking; and (III) pipe breakout (see Fig. 2b). Note that the pipeline remains more stable in a steady current than in waves for the same level of flow velocity, which is due to inertia effects for the current that are negligible compared to those for wave loading.

On the basis of flow-pipe-soil coupling modeling and dimensionless analyses, the empirical relationship between the critical Froude number $(F r)$ and dimensionless submerged weight of the pipe $(G)$ was established for describing the ocean current-induced lateral instability of shallowly embedded pipelines (Gao et al. 2007a):

$F r=a_{\mathrm{c}}+b_{\mathrm{c}} G$

where

$F r=\frac{U_{\mathrm{CL}}}{\sqrt{g D}}$
$G=\frac{W_{\mathrm{s}}}{\gamma^{\prime} D^{2}}$

where $U_{\mathrm{CL}}$ is the critical flow velocity for pipe lateral instability; $W_{\mathrm{s}}$ is the submerged weight of the pipe per unit length; 
$\gamma^{\prime}$ is the buoyant unit weight of sand; and $a_{\mathrm{c}}$ and $b_{\mathrm{c}}$ are two empirical coefficients, e.g., $a_{\mathrm{c}}=0.102, b_{\mathrm{c}}=0.423$ for the steady currents and medium sands. Note that the empirical relationship (Eq. 3) was obtained under the very shallow embedment condition (e.g., $e / D<0.05$ ), for which the passive pressure of the soil is negligible.

An analytical pipe-soil interaction model was recently developed using a limit equilibrium approach to evaluate current-induced lateral instability of a partially embedded pipeline on a sloping sandy seabed (Gao et al. 2016), which is simplified for the special case of the horizontal seabed (see Appendix A). The total lateral resistance $F_{\mathrm{R}}$ is derived with separation into two components, i.e., the passivepressure component $F_{\mathrm{Rp}}$ and the sliding-friction component $F_{\mathrm{Rf}}$ :

$$
F_{\mathrm{R}}=\underbrace{0.5 \gamma^{\prime} e^{2} K_{\mathrm{p}} \cos \varphi^{\prime}}_{F_{\mathrm{Rp}}}+\underbrace{E_{2} \sin \varphi}_{F_{\mathrm{Rf}}}
$$

where $K_{\mathrm{p}}$ is the passive pressure coefficient; $\varphi^{\prime}$ is the mobilized friction angle along the virtual retaining wall; and $E_{2}$ is the sliding-friction components (see Fig. 9, Appendix A).

Besides the above advances, a comprehensive review on pipeline-seabed interactions was published recently by Fredsøe (2016). In the existing literature, the tunnel erosion of sand and the lateral instability of the pipe for the pipeline-seabed interaction system have been investigated separately. It can be seen that in the previous tunnelerosion modeling, the pipeline was assumed to be a fixed rigid boundary, and in the lateral-instability studies, the elasto-plastic behavior of soil and the interfacial characteristics of the pipe-soil interaction were mainly taken into account. Nevertheless, the tunnel erosion of sand and the lateral instability of the pipeline should be competitive during the process of the instability in such a pipe-soil interaction system under the action of ocean currents. The question is, which has the priority to emerge for the two competitive physical phenomena. As such, we investigate the mechanism of competition between tunnel erosion and lateral instability in order to gain a better understanding of the instability of the pipe-soil interaction system.

In this study, correlation analyses are performed on the competition mechanism for the aforementioned two physical processes. The instability envelope for the pipe-soil interaction system is established by correlating the critical flow velocities for tunnel erosion and lateral instability. Parametric study is further conducted on the effects of initial embedment, the internal friction angle of sand, and the submerged weight of the pipeline.

\section{Competition between tunnel erosion and lateral instability}

\section{Critical velocity and instability envelope of the pipe-soil interaction system}

As previously mentioned, the underlying physical mechanisms of the tunnel erosion of sand and the lateral instability of pipes are different from each other. The controlling parameters of non-dimensional flow velocity for tunnel erosion and of lateral instability are the revised Shields number (Eq. 2) and the Froude number (Eq. 4a), respectively. Therefore, for the purpose of comparisons between such two processes, the critical flow velocity for the tunnel erosion $\left(U_{\mathrm{CT}}\right)$ and that for the lateral instability $\left(U_{\mathrm{CL}}\right)$ are correlated directly in this section.

In accordance with Eq. 1, the critical flow velocity for tunnel erosion can be obtained as follows:

$U_{\mathrm{CT}}=\sqrt{(2.51+0.068 \varphi)(1-n)(s-1) g e}$

As for the lateral instability of a pipeline, to evaluate the critical flow velocity $\left(U_{\mathrm{CL}}\right)$, the analytical pipe-soil interaction model by Gao et al. (2016) is adopted, which is simplified for a horizontal seabed condition. The ultimate lateral soil resistance to the pipeline under ocean currents can be expressed as follows (see Appendix A):

$$
F_{\mathrm{R}}=0.5 \gamma^{\prime} e^{2} K_{\mathrm{p}} \cos \varphi^{\prime}\left(1+\frac{\sin \varphi \sin (\beta-\delta-\omega)}{\cos \omega \cos (\beta-\delta+\varphi)}\right)
$$

where $\beta=\frac{\pi}{2}-\frac{\pi}{2} \theta, \theta$ is the half angle of pipe penetration $\theta=$ $\arccos (1-2 e / D), \omega=\arctan \left(\frac{0.5 \gamma^{\prime} e^{2} K_{\mathrm{p}} \sin \varphi^{\prime}-W_{\mathrm{b}}}{0.5 \gamma^{\prime} e^{2} K_{\mathrm{p}} \cos \varphi^{\prime}}\right), W_{\mathrm{b}}$ is the submerged weight of the carried soil wedge; and $\delta$ is the mobilized pipe-soil interfacial friction angle, the absolute values of which should be no larger than its critical value $\delta_{\text {crit. }}$. Based on the passive soil pressure theory, the friction angle $\varphi^{\prime}$ on the virtual retaining wall is always partially mobilized, whose value is generally less than $\varphi / 3$. It is assumed that a fully smooth virtual retaining wall $\left(\varphi^{\prime}=0\right)$ would be a conservative treatment for predicting the critical flow velocity (see Gao et al. 2016). Hence, the passive pressure coefficient $K_{\mathrm{p}}$ in Eq. 7 can be evaluated with the conventional Rankine's theory for passive earth pressure, i.e., $K_{\mathrm{p}}=(1+\sin \varphi) /(1-\sin \varphi)$.

As previously stated, when a pipeline subjected to ocean currents can maintain lateral stability, there normally exists a balance between hydrodynamic loads, the submerged weight of the pipe, and lateral soil resistance (see Fig. 1). As for a pipeline on a rigid bed, the drag force $F_{\mathrm{D}}$ and lift force $F_{\mathrm{L}}$ can be calculated using Morison's equations:

$$
\begin{aligned}
& F_{\mathrm{D}}=0.5 C_{\mathrm{D}} \rho_{\mathrm{w}} D U_{\mathrm{CL}}^{2} \\
& F_{\mathrm{L}}=0.5 C_{\mathrm{L}} \rho_{\mathrm{w}} D U_{\mathrm{CL}}^{2}
\end{aligned}
$$


in which the $\operatorname{drag}\left(C_{\mathrm{D}}\right)$ and lift $\left(C_{\mathrm{L}}\right)$ force coefficients can be determined by their relationships with Reynolds number $\left(\operatorname{Re}=U_{\mathrm{CL}} D / \nu\right.$, where $\nu$ is the kinematic viscosity of water $)$ (Jones 1978). Note that the hydrodynamic coefficients recommended by Jones (1978) were obtained for a pipeline just laid on the seabed surface with an embedment-to-diameter ratio of $e / D=0$, whereas the hydrodynamics were measured as varying for partially embedded pipelines, which highly depends on the embedment-to-diameter ratio of the pipeline (Jacobsen et al. 1989; Det Norske Veritas 2010; Peng et al. 2016). The variations of hydrodynamics reduction coefficients in the horizontal $\left(r_{\mathrm{emb}, \mathrm{H}}\right)$ and vertical $\left(r_{\mathrm{emb}, \mathrm{L}}\right)$ directions with $e / D$ suggested in the Det Norske Veritas (DNV) recommended practice (Det Norske Veritas 2010) are used here (see Fig. 3):

$$
r_{\mathrm{emb}, \mathrm{L}}=1.0-1.3\left(e / D^{-0.1}\right)
$$

Then, $F_{\mathrm{D}}$ and $F_{\mathrm{L}}$ in the Morison's equations can be revised as follows, taking into account hydrodynamics reduction coefficients:

$F_{\mathrm{D}}=0.5 r_{\mathrm{emb}, \mathrm{H}} C_{\mathrm{D}} \rho_{\mathrm{w}} D U_{\mathrm{CL}}^{2}$

$F_{\mathrm{L}}=0.5 r_{\mathrm{emb}, \mathrm{L}} C_{\mathrm{L}} \rho_{\mathrm{w}} D U_{\mathrm{CL}}^{2}$

where $\rho_{\mathrm{w}}$ is the density of water.

For the lateral stability of a submarine pipeline under ocean currents, the drag force $\left(F_{\mathrm{D}}\right)$ on the pipeline should be balanced with the soil lateral resistance $\left(F_{\mathrm{R}}\right)$ :

\section{$F_{\mathrm{D}}=F_{\mathrm{R}} \quad$ (in the horizontal direction)}

Meanwhile, the supporting force $\left(F_{\mathrm{C}}\right)$ of the underlying soil should be positive and be balanced with the submerged weight of the pipeline $\left(W_{\mathrm{S}}\right)$ and the lift force $\left(F_{\mathrm{L}}\right)$ :

$$
F_{\mathrm{C}}=\mathrm{W}_{\mathrm{S}}-F_{\mathrm{L}} \text { and } F_{\mathrm{C}} \geq 0 \text { (in the vertical direction) }
$$

Note that where the contacting force approaches zero $\left(F_{\mathrm{C}}\right.$ $\rightarrow 0$ ) due to the increased lift force (e.g., for light pipelines), the pipeline may break out of its original location and lose lateral stability due to the drag force. Submitting Eqs. (7, 10a) into Eq. (11a), the implicit expression of the critical flow velocity $U_{\mathrm{CL}}$ for the lateral instability of pipeline can be derived as follows:

$$
U_{\mathrm{CL}}=\sqrt{\left(1+\frac{\sin \varphi \sin (\beta-\delta-\omega)}{\cos \omega \cos (\beta-\delta+\varphi)}\right) \frac{\gamma^{\prime} e^{2} K_{\mathrm{p}}}{r_{\mathrm{emb}, \mathrm{H}} C_{\mathrm{D}} \rho_{\mathrm{w}} D}}
$$

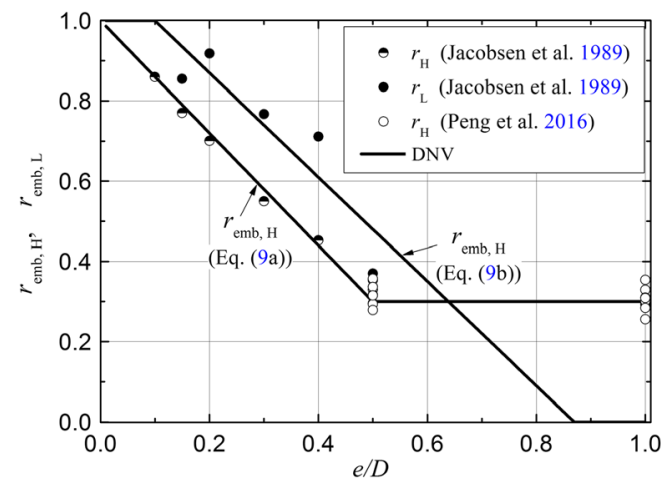

Fig. 3 Variation of the hydrodynamics reduction coefficients with embedment-to-diameter ratio $e / D$ for partially embedded pipelines. $\mathrm{r}_{\mathrm{emb}, \mathrm{H}}$ hydrodynamics reduction coefficient in the horizontal direction, $r_{\mathrm{emb}, \mathrm{L}}$ hydrodynamics reduction coefficient in the vertical direction

Note that once the value of $U_{\mathrm{CL}}$ is obtained, the force balance in the vertical direction should be checked using Eq. $11 b$.

The critical flow velocities $U_{\mathrm{CT}}$ and $U_{\mathrm{CL}}$ for the two potential instability mechanisms, i.e., tunnel erosion (termed 'mechanism-I') and lateral instability ('mechanism-II') of the submarine pipeline can be calculated with Eqs. 6 and 12, respectively. Given the properties of the sandy seabed (e.g., $\varphi=43^{\circ}, n=0.53, \gamma^{\prime}=7.6 \mathrm{kN} / \mathrm{m}^{3}$, $s=2.65)$ and the water $\left(\rho_{\mathrm{w}}=1.0 \times 10^{3} \mathrm{~kg} / \mathrm{m}^{3}, \nu=\right.$ $1.5 \times 10^{-6} \mathrm{~m}^{2} / \mathrm{s}$ ), the critical lines of flow velocities (generally denoted as $U_{\mathrm{cr}}$ ) varying with the embedment-todiameter ratio $(e / D)$ for tunnel erosion (line- $\mathrm{AB}^{\prime}$, see Fig. 4) and lateral instability (line-A'B) can be obtained, respectively, for a fixed value of the dimensionless submerged weight of pipeline $(G=0.4)$.

Note that with increasing pipeline embedment, the seepage channel (along the circumference segment) below the embedded pipeline gets enlarged, which would correspondingly increase the critical flow velocity for tunnel erosion of sand (see Eqs. 1 and 2). At the same time, the lateral soil resistance is also enhanced with increasing embedment (see Eq. 7), which would also increase the

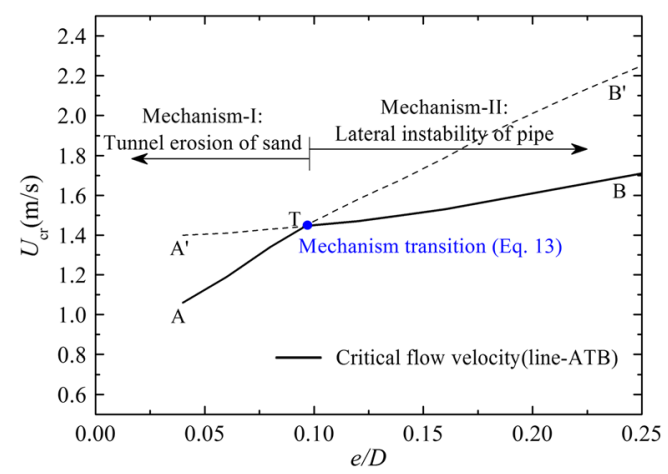

Fig. 4 Variation of critical flow velocity $U_{\text {cr }}$ with embedment-todiameter ratio $e / D$ (sand: $\varphi=43^{\circ}, n=0.53, \gamma^{\prime}=7.6 \mathrm{kN} / \mathrm{m}^{3}, s=2.65$; water: $\rho_{\mathrm{w}}=1.0 \times 10^{3} \mathrm{~kg} / \mathrm{m}^{3}, \nu=1.5 \times 10^{-6} \mathrm{~m}^{2} / \mathrm{s}$; pipe: $D=0.5 \mathrm{~m}, G=0.4$ ) 
critical flow velocity for the lateral instability of pipeline (see Eq. 12). Therefore, the two mechanisms, i.e., the tunnel erosion and lateral instability, are competitive for a partially embedded pipeline under the action of ocean currents.

Figure 4 shows that both critical velocities increase approximately linearly with increasing $e / D$, and the two critical lines (line- $\mathrm{AB}^{\prime}$ and line- $\mathrm{A}^{\prime} \mathrm{B}$ ) intersect at point-T, i.e., $(e / D)_{\mathrm{T}} \approx 0.09$, where $(e / D)_{\mathrm{T}}$ is the embedment-to-diameter ratio for transition of instability mechanisms. When the pipeline embedment $e / D$ $<(e / D)_{\mathrm{T}}$, the line segment-AT (for tunnel erosion) is lower than the line segment-A'T (for lateral instability), indicating the tunnel erosion of sand would be triggered for shallow embedment conditions. Similarly, when the pipeline embedment is further increased, i.e., $e / D \geq(e / D)_{\mathrm{T}}$, the line segment$\mathrm{TB}^{\prime}$ (for tunnel erosion) becomes higher than the line segment-TB (for lateral instability), indicating the lateral instability of pipeline would be prone to be triggered for deep embedment conditions. Such correlation analysis indicates that the critical lines for the instability of a pipe-soil interaction system should be the solid fold line-ATB, below which the system is stable (see Fig. 4).

As shown in Fig. 4, point $\mathrm{T}$ is the transition point for instability mechanisms transiting from tunnel erosion of sand to lateral instability of the pipe with the increase in pipeline embedment for a certain value of submerged weight of the pipeline. If we let $U_{\mathrm{CT}}=U_{\mathrm{CL}}$, the embedment-to-diameter ratio for transition of instability mechanisms $(e / D)_{\mathrm{T}}$ is derived by submitting Eqs. 6 and 12:

$U_{\mathrm{cr}}=\left\{\begin{array}{l}\sqrt{(1-n)\left(s^{-1}\right)(2.51+0.068 \varphi) g e} \\ \sqrt{\left(1+\frac{\sin \varphi \sin (\beta-\delta-\omega)}{\cos \omega \cos (\beta-\delta+\varphi)}\right) \frac{\gamma^{\prime} e^{2} K_{\mathrm{p}}}{r_{\mathrm{emb}, \mathrm{H}} C_{\mathrm{D}} \rho_{\mathrm{w}} D}}\end{array}\right.$

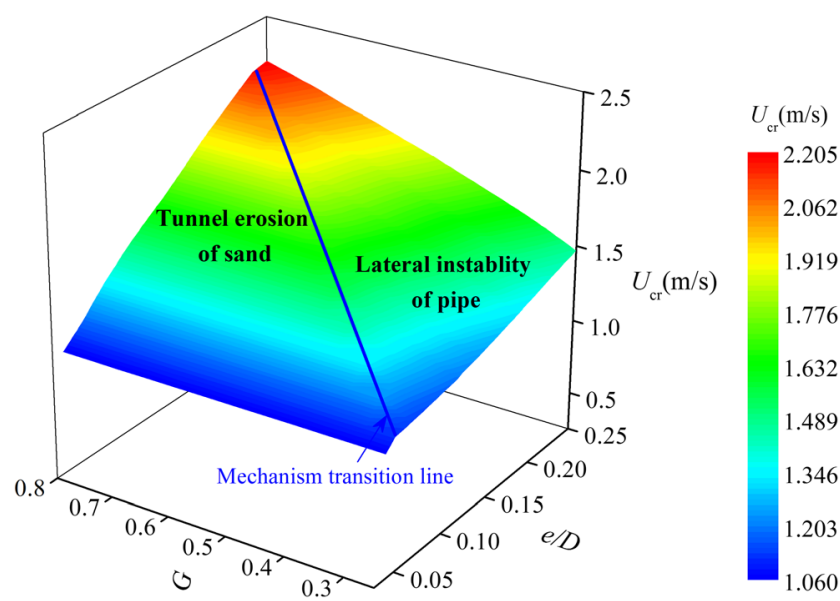

Fig. 5 Instability envelope for the pipe-soil interaction system (sand: $\varphi=43^{\circ}, n=0.53, \gamma^{\prime}=7.6 \mathrm{kN} / \mathrm{m}^{3}, s=2.65$; water: $\rho_{\mathrm{W}}=1.0 \times 10^{3} \mathrm{~kg} /$ $\mathrm{m}^{3}, \nu=1.5 \times 10^{-6} \mathrm{~m}^{2} / \mathrm{s}$; pipe: $\left.D=0.5 \mathrm{~m}\right)$. $\mathrm{U}_{\text {cr }}$ critical flow velocity

$(e / D)_{\mathrm{T}}=\frac{r_{\mathrm{emb}, \mathrm{H}} C_{\mathrm{D}}(2.51+0.068 \varphi)}{K_{\mathrm{p}}\left(1+\frac{\sin \varphi \sin (\beta-\delta-\omega)}{\cos \omega \cos (\beta-\delta+\varphi)}\right)}$

The parameters $\beta, \delta$, and $\omega$ in Eq. 13 are also the functions of $e / D$ (see Appendix A), so the values of $(e / D)_{\mathrm{T}}$ can be determined with iteration calculation. The critical flow velocity for the instability of the pipe-soil interaction system (solid fold line-ATB, see Fig. 4) can thereby be described using the following piecewise function:

for $e / D<(e / D)_{\mathrm{T}}$ (tunnel erosion)

for $e / D \geq(e / D)_{\mathrm{T}} \quad$ (lateral instability)
Equation 14 indicates that when the properties of sand and water are given, the critical flow velocity $U_{\mathrm{cr}}$ is mainly related to the embedment $(e)$ and the submerged weight $\left(W_{\mathrm{s}}\right)$ of the pipeline. By using the Buckingham $\pi$ theorem of dimensional analysis, the dimensionless forms for the parameters $e$ and $W_{\mathrm{s}}$ can be deduced as $e / D$ and $G\left(=W_{\mathrm{s}} /\left(\gamma^{\prime} D^{2}\right)\right)$, respectively. Thus, an instability envelope (see Fig. 5) for the pipe-soil interaction system can be established, which is described with three key parameters, i.e., the embedment-to-diameter ratio $e / D$, the dimensionless submerged weight of pipe $G$, and the corresponding critical flow velocity $U_{\mathrm{cr}}$.

As illustrated in Fig. 5, the established instability envelope is composed of two components: one referring to the tunnelerosion mechanism and the other to the pipeline lateral instability. In between the two components is the transition line for instability mechanisms transiting from tunnel erosion to lateral instability. If the flow-velocity value of ocean currents lies above the instability envelope for the critical flow velocity, either tunnel erosion or lateral instability could be induced. The detailed procedure for determination of critical flow velocity is given in the following section.

\section{Analysis procedure for determination of instability mechanism and critical velocity}

In the on-bottom stability design for a submarine pipeline under ocean currents, the values of the main parameters for the soil, water, and pipeline should be known in advance. These parameters include the grain's mass density $\rho_{\mathrm{s}}$, the porosity $n$, and the internal friction angle $\varphi$ of the sand; the mass density $\rho_{\mathrm{w}}$ and the 
kinematic viscosity $\nu$ of the water; and the diameter $D$ of the pipeline. As analyzed previously, the critical flow velocity $U_{\mathrm{cr}}$ is dependent on the instability mechanism and related with the two dimensionless parameters, i.e., $e / D$ and $G$. The analysis procedure for determination of the critical velocity is given using the two steps given in the following two sections (see Fig. 6).

\section{Step-I}

Calculation of the embedment-to-diameter ratio $(e / D)_{\mathrm{T}}$ for transition of instability mechanisms

The embedment-to-diameter ratio $(e / D)_{\mathrm{T}}$ can be determined using Eq. 13 through iteration calculations. Taking a trial value [denoted as $(e / D)_{\mathrm{T}}$ ] for the first iteration, the corresponding critical velocity [i.e., $\left(U_{\mathrm{cr}}\right)_{\mathrm{T}}$ ] for the transition point can be calculated using Eq. 6. With this calculated value of $\left(U_{\mathrm{cr}}\right)_{\mathrm{T}}$, the Reynolds number Re, effective hydrodynamic coefficients $\left(C_{\mathrm{D}}\right.$ and $\left.C_{\mathrm{L}}\right)$, and the resulting respective hydrodynamics $\left(F_{\mathrm{D}}\right.$ and $F_{\mathrm{L}}$, Eqs. 10a and $\left.10 \mathrm{~b}\right)$ can be determined. Once the value of the dimensionless submerged weight of the pipeline $G$ is specified, the pipe-soil interfacial friction angle $\delta$ can be calculated using Eq. A9. Then, the embedment-to-diameter ratio for the transition of instability mechanisms can be tentatively calculated using Eq. 13. Checking the relative change between the trial and calculated values, if $\left|(e / D)_{\mathrm{T}}-(e / D)_{\mathrm{T}}^{\prime}\right| /(e / D)_{\mathrm{T}}$ is larger than the permitted value (e.g., $0.1 \%)$, the trial value of $(e / D)_{\mathrm{T}}{ }^{\prime}$ will be further revised and the iteration process will be repeated to identify the embedment-to-diameter ratio $(e / D)_{\mathrm{T}}$.

Step-II Determination of instability mechanism and corresponding critical velocity

Once the value for the embedment-to-diameter ratio $e / D$ of the pipeline is provided, referring to the calculated value of $(e /$ $D)_{\mathrm{T}}$ in Step-I, the instability mechanism for the pipe-soil interaction system can be identified. That is, if $e / D<(e / D)_{\mathrm{T}}$, the tunnel erosion of sand would be prone to being triggered; otherwise, i.e., $e / D \geq(e / D)_{\mathrm{T}}$, lateral instability of the pipeline would be triggered. The corresponding critical flow velocity $U_{\text {cr }}$ for the system instability can then be calculated with Eq. 14. As indicated in Eq. 14, while calculating the critical velocity for the lateral instability, the parameters $C_{\mathrm{D}}, \delta$ are the function of $U_{\mathrm{cr}}$. As such, the determination of $U_{\mathrm{cr}}$ also needs iteration calculations, which is similar to that for the calculation of $(e / D)_{\mathrm{T}}$.

\section{Parametric study on the stability of pipe-soil interaction system}

Parametric study is performed to investigate the influence of the internal friction angle of sand and submerged weight of

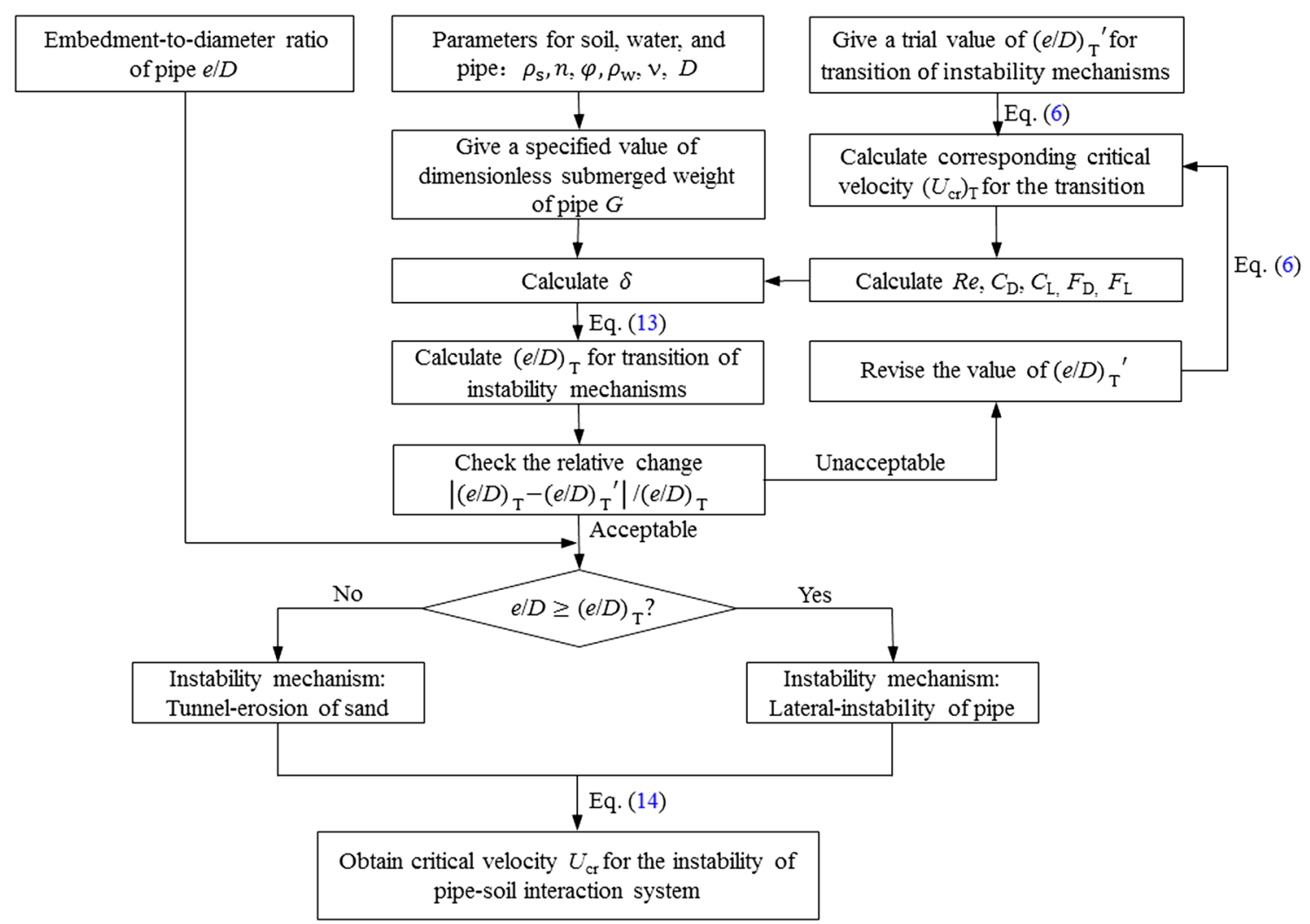

Fig. 6 Analysis procedure for determination of the instability mechanism and critical velocity for the pipe-soil interaction system. $e / D$ embedment-todiameter ratio, $(e / D)_{\mathrm{T}}$ trial value for the iteration calculations of the embedment-to-diameter ratio, $U_{\mathrm{cr}}$ critical flow velocity 
Table 1 Input data for ocean current, sand, and pipeline

\begin{tabular}{|c|c|c|}
\hline Parameters & Values & Notes \\
\hline \multicolumn{3}{|l|}{ (a) Parameters of ocean current } \\
\hline Density of water $\rho_{\mathrm{w}}\left(\mathrm{kg} / \mathrm{m}^{3}\right)$ & $1.0 \times 10^{3}$ & \\
\hline Kinematic viscosity of water $\nu\left(\mathrm{m}^{2} / \mathrm{s}\right)$ & $1.5 \times 10^{-6}$ & \\
\hline \multicolumn{3}{|l|}{ (b) Properties of sandy seabed } \\
\hline Internal friction angle of the sand $\varphi$ & $43^{\circ}, 30^{\circ}$ & Various \\
\hline Passive pressure coefficient $K_{\mathrm{p}}$ & $5.29,3.0$ & Eq. A3 \\
\hline Porosity of soil $n$ & 0.53 & \\
\hline Mass density of sand grains $\rho_{\mathrm{s}}\left(\mathrm{kg} / \mathrm{m}^{3}\right)$ & $2.65 \times 10^{3}$ & \\
\hline Specific gravity of sand grains $s$ & 2.65 & $s=\rho_{\mathrm{s}} / \rho_{\mathrm{w}}$ \\
\hline Effective unit weight of the sand $\gamma^{\prime}\left(\mathrm{kN} / \mathrm{m}^{3}\right)$ & 7.6 & $\gamma^{\prime}=(1-n)(s-1) \rho_{\mathrm{w}} g$ \\
\hline \multicolumn{3}{|l|}{ (c) Parameters of the pipeline } \\
\hline Pipe diameter $D(\mathrm{~m})$ & 0.5 & \\
\hline Embedment-to-diameter ratio $e / D$ & $0.05 \sim 0.25$ & Various \\
\hline Submerged weight of pipe $W_{\mathrm{s}}(\mathrm{kN} / \mathrm{m})$ & $0.475 \sim 1.615$ & Various \\
\hline Dimensionless submerged weight of pipe $G$ & $0.25 \sim 0.85$ & Various \\
\hline
\end{tabular}

the pipe on the stability of pipe-soil interaction system. Table 1 lists the input data for the main parameters of ocean current, sandy seabed, and pipeline, respectively. When the values of these parameters are known, the instability mechanisms and their corresponding critical velocities can be evaluated according to the analysis procedure given earlier, as shown in Fig. 6.

\section{Effect of the internal friction angle of sand}

Figure 7a gives the results of the relations between the critical flow velocity $U_{\text {cr }}$ and internal friction angle of sand $\varphi$ (termed ' $U_{\mathrm{cr}} \sim \varphi$ relationship'). For a certain value of $e / D$, with the increase of $\varphi$, the critical flow velocity for tunnel erosion and for lateral instability increase. Moreover, to investigate the influence of the internal friction angle on the embedment-to-diameter ratio $(e / D)_{\mathrm{T}}$ for transition of the instability mechanism, Fig. $7 \mathrm{~b}$ gives variations of the critical flow velocity $U_{\text {cr }}$ with the embedment-to-diameter ratio e/ $D$ (termed ' $U_{\text {cr }} \sim e / D$ relationship') for two typical values of the internal friction angle of sand $\varphi$, i.e., $\varphi=30^{\circ}$ (loose sand) and $43^{\circ}$ (dense sand). Note that the values of $\varphi$ can be evaluated with the concept of the relative dilatancy index (Bolton 1986), i.e., for a plane strain problem $\varphi \approx \varphi_{\text {crit }}+$ $5 I_{\mathrm{R}}$, where $\varphi_{\text {crit }}$ is the critical state angle of shearing resistance of sands, depending on the ingredients of the grains; $I_{\mathrm{R}}$ is the relative dilatancy index $I_{\mathrm{R}}=D_{\mathrm{r}}\left(10-\ln p^{\prime}\right)-1$, in which $D_{\mathrm{r}}$ is the relative density of sands and $p$ is the mean effective stress (in $\mathrm{kPa}$ ). As shown in Fig. 7b, the embedment-to-diameter ratio $(e / D)_{\mathrm{T}}$ for transition of instability mechanisms is also increased according to the increase of $\varphi$. The competition mechanism implies that the effects of internal friction angle on lateral instability are more remarkable than on tunnel erosion.

When the variation of hydrodynamics reduction coefficients (Eqs. 9a and 9b) is considered, Fig. 7b shows that the effects of hydrodynamics reduction due to pipe embedment on the critical flow velocity becomes more obvious with the increasing embedment-to-diameter ratio. As such, if the hydrodynamics reduction is neglected, the on-bottom stability of the pipe-soil interaction system would be underestimated.

\section{Effect of the submerged weight of the pipeline}

As illustrated in Fig. 5, given the fundamental properties of soil, water, and the pipeline, the established instability envelope can be described using the embedment-to-diameter ratio $(e / D)$, the dimensionless submerged weight of the pipeline $(G)$, and the corresponding critical flow velocity $\left(U_{\mathrm{cr}}\right)$. To reveal the effects of the submerged weight of the pipeline in detail, Fig. 8 gives the $U_{\mathrm{cr}} \sim e / D$ relationships for various values of $G$.

The two series of $U_{\mathrm{cr}} \sim e / D$ relationships in Fig. 8 show that the effects of the submerged weight of the pipeline are quite significant. The embedment-to-diameter ratio (e) $D)_{\mathrm{T}}$ for transition of instability mechanisms generally increases with an increase in the submerged weight of pipeline $G$. It should be noted that for light pipelines (e.g., $G<0.2$ ), only lateral instability could be induced for the examined range of pipeline embedment (for such cases, the values of $e / D$ are normally lower than those for heavier pipelines). Because the competition mechanism always exists between tunnel erosion and lateral 


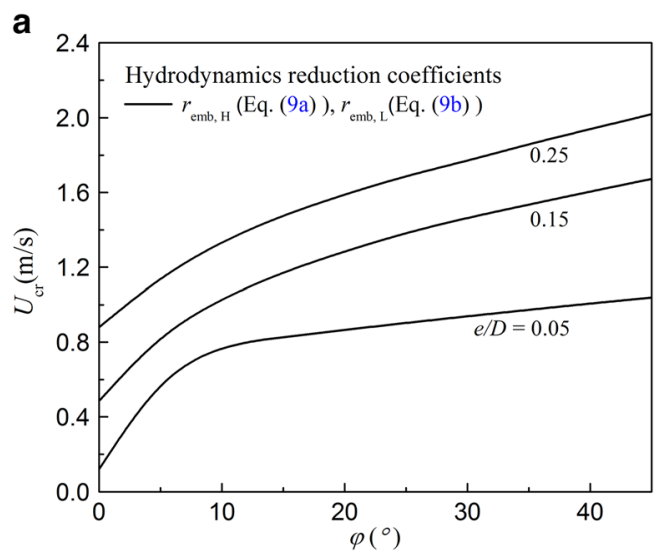

b

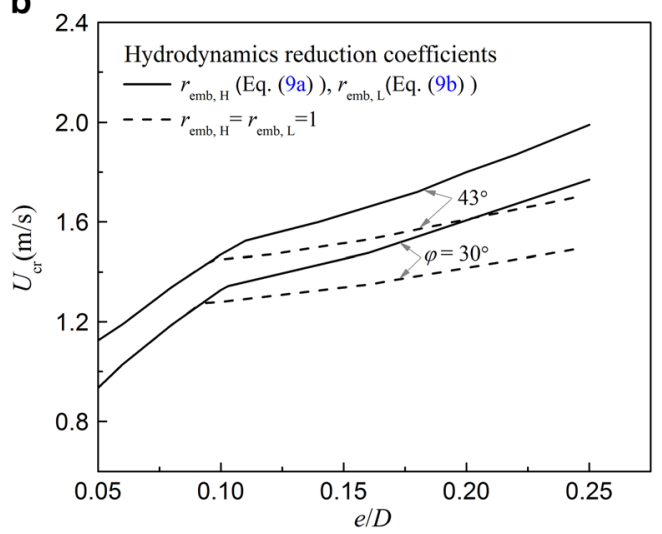

Fig. 7 Effect of internal friction angle: (a) $U_{\mathrm{cr}} \sim \varphi$ relationships; (b) $U_{\text {cr }} \sim e / D$ relationships for 2 typical values of $\varphi$; hydrodynamics reduction due to pipe embedment is also considered $(G=0.4$; the values of other parameters are given in Table 1). e/D embedment-todiameter ratio, $r_{\mathrm{emb}}$, hydrodynamics reduction coefficient in the horizontal direction, $r_{\mathrm{emb}}$, $\mathrm{L}$ hydrodynamics reduction coefficient in the vertical direction, $U_{\mathrm{cr}}$ critical flow velocity

instability, it is indicated that, for a given value of $e / D$, lateral instability is more likely to be triggered than tunnel erosion with a decrease in $G$.

\section{Conclusions}

In previous studies on pipe-soil interactions, the tunnel erosion and lateral instability of submarine pipelines were investigated separately. As such, two phenomena could be triggered alternatively in submarine geological and hydrodynamic environments, correlation analyses are performed in this study to reveal the underlying competition mechanism. The following conclusions can be drawn:

(1) The critical flow velocities of ocean currents for tunnel erosion and lateral instability for a partially embedded pipeline are derived via correlation analyses. The instability envelope is then established, which is described using three key parameters: the

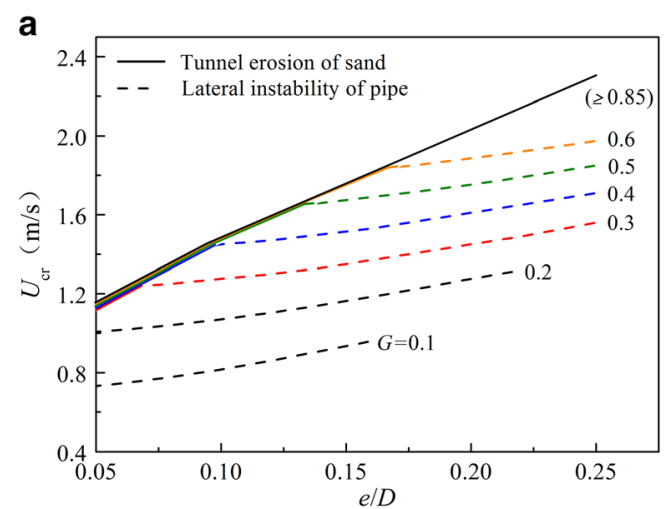

b

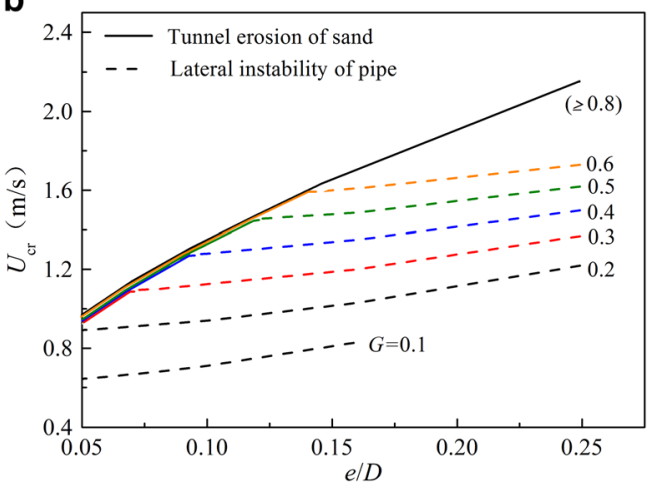

Fig. 8 Effect of dimensionless submerged weight of pipeline: $U_{\mathrm{cr}} \sim e / D$ relationships for various values of $G$ : (a) $\varphi=43^{\circ}$; (b) $\varphi=30^{\circ}$ (the values of other parameters are given in Table 1$) . e / D$ embedment-to-diameter ratio, $U_{\text {cr }}$ critical flow velocity

embedment-to-diameter ratio, the dimensionless submerged weight of the pipe, and the corresponding critical flow velocity.

(2) Parametric study indicates that the critical flow velocity increases with an increasing internal friction angle of sand. The effects of the hydrodynamics reduction due to pipe embedment on critical flow velocity become more obvious for larger values of the embedment-todiameter ratio.

(3) Tunnel erosion is more prone to emerge than lateral instability for shallowly embedded pipelines. With an increasing embedment-to-diameter ratio, tunnel erosion could be suppressed and the lateral instability then becomes easier to induce. With a decrease in the dimensionless submerged weight of the pipeline, the lateral instability is more likely to be triggered than the tunnel erosion for a given value of the embedment-to-diameter ratio.

Acknowledgements This work is financially supported by the National Natural Science Foundation of China (Grant Nos. 11372319; 11232012) and the Strategic Priority Research Program (Type-B) of CAS (Grant No. XDB22030000). Helpful discussions with Dr. Wen-Gang Qi and Mr. Jun Liu are greatly appreciated. 


\section{Appendix A: Pipe-soil interaction model for current-induced pipeline instability on a horizontal sandy seabed}

The pipe-soil interaction model proposed by Gao et al. (2016) was derived by using a limit equilibrium approach for predicting ultimate lateral soil resistance to the partially embedded pipeline on a sloping sandy seabed in ocean currents. For the special case of a horizontal sandy seabed (i.e., the slope angle of the seabed is zero), the model can be simplified as follows.

As illustrated in Fig. 9a, the composite failure surface for the lateral pipe-soil interaction is comprised of the sliding- friction segment-DB and the passive-pressure segment-BC. $A$ virtual retaining wall- $A B$ is supposed to be perpendicular to the seabed surface. The carried soil wedge-ABD at failure (the shaded areas, see Fig. 9a) should be chosen as the analysis object. The main forces acting on the soil wedge-ABD include four components: the passive earth pressure $\left(E_{1}\right)$ on the virtual retaining wall- $\mathrm{AB}$, the sliding-friction $\left(E_{2}\right)$ on the segment$\mathrm{DB}$, the submerged weight $\left(W_{\mathrm{b}}\right)$ of the wedge-ABD, and the pipe-soil interfacial force $(P)$. The corresponding triangle of these forces is shown in Fig. 9b.

According to the geometric principle of the composition of forces, the pipe-soil interfacial force $P$ is balanced by the resultant force of $E_{1}, E_{2}$, and $W_{\mathrm{b}}$. As the horizontal component
Fig. 9 Lateral instability of a pipeline: (a) geometry of failure mechanism; (b) triangle of the forces on the wedge-ABD [shaded area in (a)] a

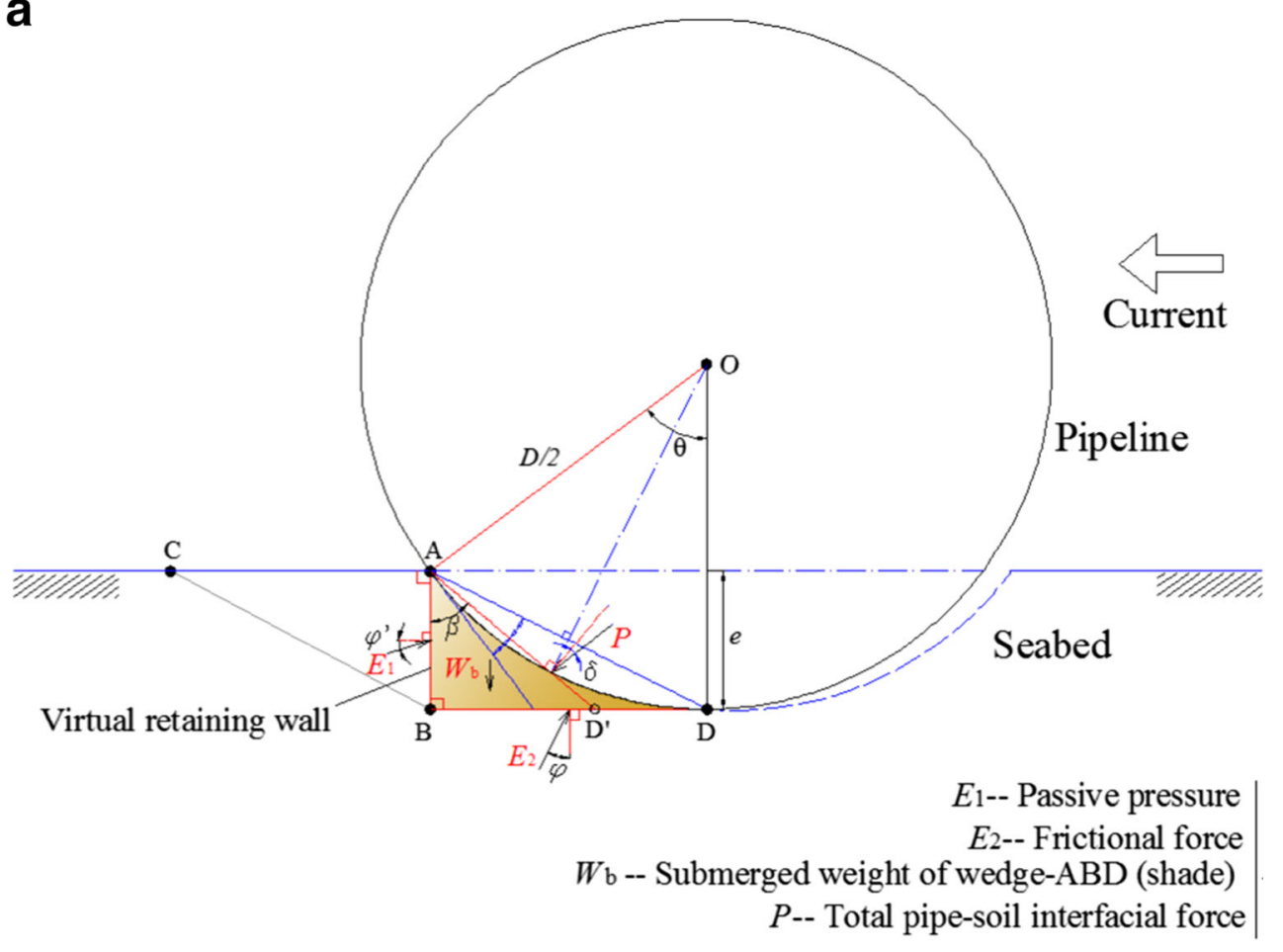

b

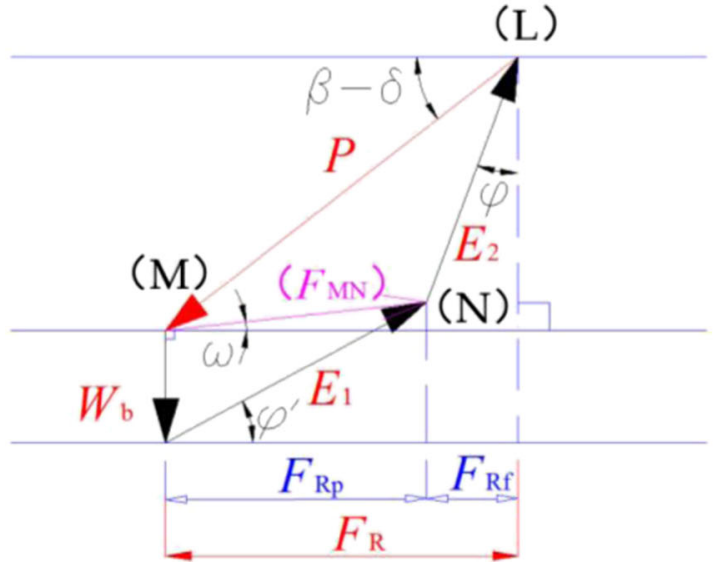


of the interfacial force $P$, the lateral soil resistance $F_{\mathrm{R}}$ can then be divided into the passive pressure $\left(F_{\mathrm{Rp}}\right)$ and the sliding friction $\left(F_{\mathrm{Rf}}\right)$ components, respectively:

$$
F_{\mathrm{R}}=\underbrace{E_{1} \cos \varphi^{\prime}}_{F_{\mathrm{Rp}}}+\underbrace{E_{2} \sin \varphi}_{F_{\mathrm{Rf}}}
$$

where $\varphi^{\prime}$ is the mobilized friction angle along the retaining wall- $\mathrm{AB}$ and $\varphi$ is the internal friction of the sand. Based on the Coulomb's theory of passive earth pressure, the passive earth pressure $E_{1}$ can be obtained as follows:

$E_{1}=\frac{1}{2} \gamma^{\prime} e^{2} K_{\mathrm{p}}$

where $\gamma^{\prime}$ is the buoyant unit weight of sand, $e$ is the initial embedment of pipeline, and $K_{\mathrm{p}}$ is the passive pressure coefficient:

$K_{\mathrm{p}}=\left[\frac{\cos \varphi}{\sqrt{\cos \varphi^{\prime}}-\sqrt{\sin \left(\varphi+\varphi^{\prime}\right) \sin \varphi}}\right]^{2}$

In accordance with the 'law of sines' for the forces triangle (see Fig. 9b), the total sliding friction $E_{2}$ in Eq. A1 can be determined as follows:

$E_{2}=\frac{\cos \varphi^{\prime} \sin (\beta-\delta-\omega)}{\cos \omega \cos (\beta-\delta+\varphi)} E_{1}$

where $\beta$ is the intersection angle between wall- $\mathrm{AB}$ and the line segment $\mathrm{AD}^{\prime}$ (Fig. 9a) and $\omega$ is the intersection angle between the direction of $F_{\mathrm{MN}}$ to the seabed surface (Fig. 9b):

$\beta=\frac{\pi}{2}-\frac{3}{4} \theta$

$\omega=\arctan \left(\frac{E_{1} \sin \varphi^{\prime}-W_{\mathrm{b}}}{E_{1} \cos \varphi^{\prime}}\right)$

The submerged weight $W_{\mathrm{b}}$ of the soil wedge in Eq. A6 can be calculated as follows:

$W_{\mathrm{b}}=\frac{\gamma^{\prime}}{8}\left[4 e^{2} \frac{1+\cos \theta}{\sin \theta}-D^{2}(\theta-\sin \theta)\right]$

where $D$ is the diameter of pipeline and $\theta$ is the half angle of the pipeline penetration:

$\theta=\arccos (1-2 e / D)$

In Eq. A4, $\delta$ is the mobilized pipe-soil interfacial friction angle, which is relative to the ocean current-induced drag force $\left(F_{\mathrm{D}}\right)$, lift force $\left(F_{\mathrm{L}}\right)$, the submerged weight of the pipeline $\left(W_{\mathrm{S}}\right)$, and the half angle of the pipeline penetration $(\theta)$ :

$\delta=\arctan \left(\frac{F_{\mathrm{D}}}{W_{\mathrm{S}}-F_{\mathrm{L}}}\right)-\frac{3}{4} \theta$

Note that the absolute values of $\delta$ should be no larger than its critical value $\delta_{\text {crit }}$ (i.e., $|\delta| \leq \delta_{\text {crit }}$, where $\delta_{\text {crit }}=\arctan [(\sin \varphi$ $\left.\left.\cos \nu_{\mathrm{s}}\right) /\left(1-\sin \varphi \sin \nu_{\mathrm{s}}\right)\right], \nu_{\mathrm{s}}$ is the angle of soil dilation $)$.

Submitting Eqs. A2 and A4 into Eq. A1, the lateral soil resistance $F_{\mathrm{R}}$ can be re-expressed as follows:

$F_{\mathrm{R}}=0.5 \gamma^{\prime} e^{2} K_{\mathrm{p}} \cos \varphi^{\prime}\left(1+\frac{\sin \varphi \sin (\beta-\delta-\omega)}{\cos \omega \cos (\beta-\delta+\varphi)}\right)$

$A_{0.5}$, One half the area of a vertical cross-section of the soil displaced by the partially embedded pipe during penetration and oscillations (see Wagner et al. 1989); $C_{\mathrm{D}}$, Drag force coefficient; $C_{\mathrm{L}}$, Lift force coefficient; $D$, Pipe diameter; $E$, Initial embedment of pipe; $(e / D)_{\mathrm{T}}$, Embedment-to-diameter ratio for transition of instability mechanisms; $(e / D)_{\mathrm{T}}{ }^{\prime}$, Trial value for the iteration calculations of $(e / D)_{T} ; E_{1}$, Passive earth passive in the pipe-soil model by Gao et al. (2016) (see Fig. 9); $E_{2}$, Total sliding friction on a failure surface (see Fig. 9); $F_{\mathrm{L}}$, Lift force on the pipe; $F_{\mathrm{D}}$, Drag force on the pipe; $F_{\mathrm{R}}$, Lateral soil resistance to pipe; $F_{\mathrm{Rp}}$, Passive pressure component of lateral soil resistance; $F_{\mathrm{Rf}}$, Sliding friction component of lateral soil resistance; $F_{\mathrm{Rw}}$, Additional submerged weight component of lateral soil resistance; $F_{\mathrm{C}}$, Pro force of seabed to pipe; $F r$, Froude number, $U / \sqrt{g D} ; G$, Gravitational acceleration;

$G$, Dimensionless submerged weight of pipe, $W_{\mathrm{s}} /\left(\gamma^{\prime} D^{2}\right) ; I_{\mathrm{R}}$, Relative dilatancy index; $i_{\mathrm{cr} 0}$, Conventional critical hydraulic gradient for seepage failure (upward) $i_{\mathrm{cr} 0}=(1-n)(s-1) ; i_{\mathrm{cr}}$, Critical hydraulic gradient for the oblique seepage failure $i_{\mathrm{cr}}=(\sin \theta+\cos \theta \tan \varphi)(1-n)(s-1) ; K_{\mathrm{p}}$, Passive pressure coefficient; $n$, Porosity of soil;

$p$, Mean effective stress (in $\mathrm{kPa}$ ); $P$, Total pipe-soil interfacial force (see Fig. 9); Re, Reynolds number, $U D / \nu ; r_{\mathrm{emb}, \mathrm{H}}$, Reduction coefficients in the horizontal direction;

$r_{\mathrm{emb}, \mathrm{L}}$, Reduction coefficients in the vertical direction; $s$, Specific gravity of sand grains, $\rho_{\mathrm{s}} / \rho_{\mathrm{w}} ; U$, Current velocity; $U_{\mathrm{CT}}$, Critical velocity for tunnel erosion of sand; $U_{\mathrm{CL}}$, Critical velocity for lateral instability of pipe; $U_{\mathrm{cr}}$, Critical velocity for the instability of pipe-soil interaction system; $\left(U_{\mathrm{cr}}\right)_{\mathrm{T}}$, Critical velocity for transition of instability mechanisms;

$W_{\mathrm{s}}$, Submerged weight of pipe per unit length; $W_{\mathrm{b}}$, Submerged weight of the soil wedge (see Fig. 9); $\alpha$, Slope angle of seabed surface; $\beta$, Empirical coefficient in the pipesoil interaction model by Wagner et al. (1989); $\beta, \omega$, Intersection angle (Fig. 9); $\gamma$, Effective unit weight of sand, $\gamma^{\prime}=(1-n)(s-1) \rho_{\mathrm{w}} g ; \theta$, Half angle of pipe penetration; $\theta_{\mathrm{cr}}$, Non-dimensional flow velocity for the onset of tunnel erosion; 
$\mu$, Coefficient of sliding friction; $\nu$, Kinematic viscosity of water; $\nu_{\mathrm{s}}$, Angle of soil dilation; $\rho_{\mathrm{s}}$, Mass density of sand grains; $\rho_{\mathrm{w}}$, Mass density of water; $\varphi$, Internal friction angle of sand; $\varphi_{\text {crit }}$, Critical state angle of shearing resistance of sands; $\varphi$, Mobilized friction angle along retaining wall (see Fig. 9); $\delta$, Pipe-soil interfacial friction angle

\section{References}

Bolton MD (1986) The strength and dilatancy of sands. Géotechnique 36(1):65-78

Chiew YM (1990) Mechanics of local scour around submarine pipelines. J Hydraul Eng 116(4):515-529

Det Norske Veritas (2006) Free Spanning Pipelines, DNV Recommended Practice DNV-RP-F105

Det Norske Veritas (2010) On-Bottom Stability Design of Submarine Pipelines, DNV Recommended Practice DNV-RP-F109

Fredsøe J (2016) Pipeline-seabed interaction. J Waterw Port Coast Ocean Eng 142(6):03116002

Gao FP, Gu XY, Jeng DS et al (2002) An experimental study for waveinduced instability of pipelines: the breakout of pipelines. Appl Ocean Res 24(2):83-90

Gao FP, Yang B, Yan SM, Wu YX (2007b) Occurrence of spanning of a submarine pipeline with initial embedment. In: Proceedings 6th International Offshore and Polar Engineering Conference, Lisbon, Portugal, July 1-6, 2007

Gao FP, Yan SM, Yang B, Wu YX (2007a) Ocean currents-induced pipeline lateral stability on sandy seabed. J Eng Mech 133(10): 1086-1092

Gao FP, Luo CC (2010) Flow-pipe-seepage coupling analysis of spanning initiation of a partially-embedded pipeline. J Hydrodyn 22(4):478487
Gao FP, Wang N, Li JH, Han XT (2016) Pipe-soil interaction model for current-induced pipeline instability on a sloping sandy seabed. Can Geotech J 53(11)

Jones WT (1978) On-bottom pipeline stability in steady water currents. J Pet Technol 30(3):475-484

Jacobsen V, Bryndum MB, Bonde C (1989) Fluid loads on pipelines: sheltered or sliding. In: Proceedings of the 21 th Offshore Technology Conference, Houston, Texas, OTC 6056

Lyons CG (1973) Soil resistance to lateral sliding of marine pipeline. In: Proceedings 5th Annual Offshore Technology Conference, Houston, Texas, OTC 1876, pp. 479-484

Mao Y (1988) Seabed scour under pipelines. In: Proceedings 7th International Conference on Offshore Mechanics and Arctic Engineering, New York, p 33-38

Peng XF, Qi WG, Gao FP (2016) Seabed proximity effects on the waveinduced hydrodynamic forces on a submarine pipeline. In: Proceedings 12th International Society of Offshore and Polar Engineers, Gold Coast, Australia, October 4-7, 2016

Sumer BM, Fredsøe J (2002) The mechanics of scour in the marine environment. World scientific, Singapore

Sumer BM, Truelsen C, Sichmann T, Fredsøe J (2001) Onset of scour below pipelines and self-burial. Coast Eng 42(4):313-335

Wagner DA, Murff JD, Brennodden H, Svegen O (1989) Pipe-soil interaction model. J Waterw Port Coast Ocean Eng 115(2):205-220

Wang L, Liu R (2016) The effect of a berm on the lateral resistance of a shallow pipeline buried in sand. Ocean Eng 121:13-23

White DJ, Cheuk CY (2008) Modelling the soil resistance on seabed pipelines during large cycles of lateral movement. Mar Struct 21(1):59-79

Youssef BS, Tian YH, Cassidy MJ (2013) Centrifuge modelling of an onbottom pipeline under equivalent wave and current loading. Appl Ocean Res 40(2):14-25

Zang ZP, Cheng L, Zhao M et al (2009) A numerical model for onset of scour below offshore pipelines. Coast Eng 56(4):458-466

Zhang Q, Draper S, Cheng L, An H (2016) Effect of limited sediment supply on sedimentation and the onset of tunnel scour below subsea pipelines. Coast Eng 116:103-117 